war die trotzkistische Splitterpartei keineswegs nur ein politischer Kontrahent. Gegen die POUM richtete sich der Vorwurf der »fünften Kolonne«, d. h. dem »Verräter«, dem Feind in den eigenen Reihen, sodass sie zur »Agentur der Gestapo« erklärt wurde. Der POUM-Vorsitzende Andres Nin wurde daraufhin in einem Gefängnis der GPU gefoltert und umgebracht. Der in den eigenen Reihen tobende Krieg hat nach Einschätzung Michael Rohrwassers eine schwer zu quantifizierende Zahl von Toten zur Folge gehabt, sodass er die vom sowjetischen Historiker Roy Medwedew gemachte Einschätzung, dass Stalin womöglich mehr Spanienkämpfer habe erschießen lassen, als durch die Kugeln der Faschisten in Spanien gefallen sind, anzweifelt. ${ }^{76}$

Zweifelsohne wurden die schrecklichen Erfahrungen des Spanienkrieges - die Franco-Truppen vor sich und die in den eigenen Reihen operierende stalinistische Terrormaschine hinter sich - zum festen Bestandteil des historischen Gedächtnisses der noch am Leben gebliebenen antistalinistischen Linken. Die einer »traumatischen Erfahrung« (Rohrwasser) gleichkommenden Erlebnisse brachte am eindrücklichsten der Trotzkist Victor Serge 1936 in einer Frage zum Ausdruck. In einem Brief an André Gide schriebt er: »Wir Bekämpfen den Faschismus. Wie aber können wir ihm mit so vielen Konzentrationslagern im Rücken den Weg verstellen? « ${ }^{77}$ Die totalitären Erfahrungen versuchten die Renegaten literarisch aufzuarbeiten und ihren »Bruch mit dem Kommunismus $«^{78}$ zu verarbeiten. Franz Borkenau, George Orwell, Arthur Koestler, El Campesino (d.i. Valentin Gonzales), Julián Gorkin, Alfred Kantorowicz, André Malraux, Gustav Regler oder Stephen Spender weigerten sich mit ihren autobiografischen Berichten, in die kommunistische Interpretations-»Falle des Entweder-Oder « hineinzutappen und in dem Dilemma gefangen zu bleiben: »Treue zu Stalin oder Faschismus, ein Drittes gibt es nicht.«Sie erheben »per se Einspruch gegen das Postulat dieser Alternative ${ }^{79}$.

\title{
2.1 Die Erfahrungen von Margarete Buber-Neumann als exemplarischer Fall
}

Exemplarisch für die gemachten totalitären Erfahrungen mit dem kommunistischen und nationalsozialistischen Totalitarismus stehen die autobiografischen Zeugnisse von Margarete Buber-Neumann. Insbesondere die konkreten individuellen Erlebnisse nach ihrer politischen Emigration aus Deutschland in die Sowjetunion im Kontext des »Großen Terrors « in den 1930er-Jahren stehen stellvertretend für die Erfahrungen vieler ehemaliger Kommunisten und späteren Mitarbeitern des Monat mit der Herrschafts- und Verfolgungspraxis des stalinistischen Regimes.

heimschrift. Bericht eines Lebens, München 1955. Ein zweiter Auszug erschien in Der Monat 7 (1955), H. 79 (1955), S. 40-56. Koestler schilderte in den beiden Teilen seine Erfahrungen im Spanischen Bürgerkrieg. Nach der Verhaftung durch die Falangisten wurde er aufgrund internationalen Drucks wieder freigelassen. Insbesondere infolge seiner Erfahrungen in der Todeszelle des spanischen Gefängnisses brach Koestler nach seiner Befreiung mit der Kommunistischen Partei und erklärt seinen Austritt. Siehe zu Arthur Koestler auch das Porträt in Kap. II.4.3.

76 Rohrwasser, Der Stalinismus und die Renegaten, S. 66.

77 Zit. n. Victor Serge, Erinnerungen eines Revolutionärs 1901-1941, Hamburg 1977, S. 460. Die französische Ausgabe erschien 1951 unter dem Titel Mémoires d'un Révolutionnaire 1901-1941.

78 Kuhn, Bruch mit dem Kommunismus.

79 Rohrwasser, Der Stalinismus und die Renegaten, S. 1. 
Unter der Überschrift »Zwischen Rot und Braun« unterstrich Borkenau in seinem Beitrag für den Monat die sich bereits durch den Titel Als Gefangene bei Stalin und Hitler ergebende zentrale Bedeutung des Buches der ehemaligen deutschen Kommunistin Buber-Neumann. "Denn hiermit besitzen wir zum ersten Male«, so der Rezensent, »die Darstellung eines Menschen der zuerst in Händen der kommunistischen und dann der nationalsozialistischen Geheimpolizei war. Zum ersten Male können wir vergleichen, und es ist ein sehr lehrreicher Vergleich. ${ }^{80}$ Indes soll an dieser Stelle nicht der explizite Vergleich zwischen dem sowjetischen und nationalsozialistischen Herrschaftssystem im Mittelpunkt stehen, sondern ausschließlich die persönlichen Erfahrungen, die "greuelvolle[n] Erlebnisse unter zwei verschiedenen Terrorregimen ${ }^{81} \mathrm{der}$ ehemaligen Kommunistin. Buber-Neumann wurde 1901 in Potsdam geboren und trat mit 20 Jahren in den Kommunistischen Jugendverband Deutschland ein und 1926 in die KPD. In der Zeit von 1928 bis 1932 war sie bei der kommunistischen Berliner Internationalen Pressekorrespondenz, der Inprekorr, aktive Mitarbeiterin. Nach der Scheidung von Rafael Buber lernte sie 1928 ihren späteren Ehemann Heinz Neumann ${ }^{82}$ kennen, der in den 1920er-Jahren führender Funktionär der KPD war und erheblichen Anteil an der »Bolschewisierung« der Partei hatte. ${ }^{83}$ Nach der nationalsozialistischen Machteroberung 1933 emigrierte Buber-Neumann in die Sowjetunion und traf dort 1935 wieder auf Neumann.

Im »Hotel Lux«, dem Gemeinschaftshaus der Komintern und dem neuen Domizil zahlreicher ausländischer »Politemigranten«, wurde Buber-Neumann allmählich der »Terrorrealität« gewahr. Die als überzeugte Kommunistin nach Moskau Gereiste musste schockartig erleben, wie der nach dem Kirow-Mord einsetzende NKWD-Terror die gesamte sowjetische Gesellschaft betraf und die um sich greifende Parteisäuberung auch vor führenden kommunistischen Funktionären nicht haltmachte. Bereits vor dem ersten Moskauer Schauprozess im August 1936, bei dem exponierte Kommunisten wie Sinowjew oder Kamenew öffentlich gestanden, die »phantastischsten « Verbrechen begangen zu haben, und der für Buber-Neumann »zu den schlimmen Erin-

80 Franz Borkenau, Zwischen Rot und Braun, in: Der Monat 1 (1949), H. 6, S. 95-97, hier S. 95 (Rezension).

81 Ebd., S. 95. Einige Jahre später hieß es zu der Bedeutung des Buches in den einleitenden Worten der Redaktion des Monat zur Veröffentlichung des Beitrages: Margarete Buber-Neumann, Porträt eines Funktionärs. Heinz Neumanns Schicksal in Deutschland, China und Sowjetrussland, in: Der Monat 9 (1957), H. 103, S. 44-66, auf S. 44: dass »es eines der ersten authentischen Dokumente über das Schreckensregime der Säuberungen (war), über die Zwangsarbeitslager jenseits des Urals und über den perfiden Handel mit dem die Sowjets in der ersten Hälfte des Krieges einige deutsche Kommunisten, die seit 1933 bei ihnen Zuflucht gesucht hatten, aber nicht linientreu genug gewesen waren, der Gestapo in die Hände lieferten«.

82 Heinz Neumann (1902-1937) trat 1919 in die KPD ein. Sein Aufstieg im Parteiapparat erfolgte vor allem in den späten 1920er-Jahren. 1929 wurde er Mitglied des Zentralkomitees und Kandidat des Politbüros der KPD. 1932 ging Neumann aus innerparteilichen Fraktions- und Cliquenkämpfen als Verlierer heraus und wurde von allen Funktionen entbunden. Zu Beginn der 1930er-Jahre wurde Neumann als Kominternmitarbeiter nach Spanien geschickt. Nach einer Verhaftung 1934 in der Schweiz ging er dann 1935 in die Sowjetunion. Siehe hierzu im Einzelnen den bereits genannten Beitrag: Margarete Buber-Neumann, Porträt eines Funktionärs. Heinz Neumanns Schicksal in Deutschland, China und Sowjetrußland, in: Der Monat 9 (1957), H. 103, S. 44-66 (hierbei handelte es sich um einen Auszug aus ihrem Buch Von Potsdam nach Moskau. Stationen eines Irrweges [Stuttgart 1957]).

83 Vgl. Heinz Neumann, Was ist Bolschewisierung?, Hamburg 1925. 
nerungen « gehörte, ${ }^{84}$ erlebte sie, wie die Tschistka, d.h. die Parteisäuberung, auch unter den ausländischen Kommunisten im Moskauer Exil wütete. In Angst und Schrecken wurde sie versetzt, als sie feststellen musste, dass der stalinistische Terror immer mehr Freunde aus den kommunistischen Parteien erfasste. Einen »Schock« löste beispielsweise die bei »Nacht und Nebel« durchgeführte Verhaftung des sogenannten Versöhnlers Heinrich Süßkind aus. ${ }^{85}$ Mit Willi Münzenberg wurde sogar der Lebensgefährte ihrer Schwester Babette ${ }^{86}$ Opfer des institutionellen Terrors. ${ }^{87}$ Zudem musste sie miterleben, wie im Laufe der Zeit einzelne kommunistische Funktionäre aus Angst vor der Verhaftung durch den NKWD Selbstmord begingen. ${ }^{88}$

Die totalitären Bedingungen des Stalinismus und speziell der Terror des sowjetischen Geheimdienstes zeitigte Konsequenzen, die bis in die "Privatsphäre« von Buber-Neumann hineinreichten. Hierzu schrieb sie in ihrem autobiografischen Erinnerungsbericht Als Gefangene bei Stalin und Hitler:

In Moskau war die Atmosphäre zum Ersticken. Ehemalige politische Freunde wagten nicht mehr, einander zu besuchen. Das Hotel »Lux« konnte man nur mit einem Durchlaßschein betreten. Jeder Besucher wurde registriert. Dadurch hatte die NKWD eine vorzügliche Kontrolle. Die Telefone in den einzelnen Zimmern des Hotels wurden überwacht. Immer wieder bemerkten wir das knackende Ceräusch, nachdem die Verbindung hergestellt war. Die Post unterlag selbstverständlich einer Kontrolle. - Die Furcht vor Bespitzelung nahm solche Formen an, daß gute Freunde, wenn sie es doch gewagt hatten, zu Besuch zu kommen, sich zuflüsterten: »Habt ihr euer Zimmer auch genau durchsucht, ob man nicht irgendwo einen Abhörapparat einmontiert hat? Ist nicht vielleicht irgendwo ein Mikrofon angebracht? Etwa in der Lampe? Vielleicht im Telefon? ${ }^{89}$

$\mathrm{Zu}$ den schrecklichen Erfahrungen, die Buber-Neumann im »Lux« unter den prominenten kommunistischen »Politemigranten« machte, zählte auch die Tatsache, dass der »von oben« in Gang gebrachte Terror des sowjetischen Regimes auf tätige Mithilfe »von unten « rechnen konnte. Denn in der »Moskauer Menschenfalle « ${ }^{90}$ wurde in tragischer Verschränkung auch das Opfer zum »Täter«. Buber-Neumann musste erleben, wie unter den Bedingungen der stalinistischen Säuberungen auch unter den KPD-Mitgliedern im Moskauer »Lux« die Bereitschaft zur Denunziation angestachelt

84 Margarete Buber-Neumann, Von Potsdam nach Moskau. Stationen eines Irrweges, Köln-Lövenich 1981, S. 425.

85 Siehe ebd., S. $425 \mathrm{f}$.

86 Bevor Buber-Neumanns Schwester Babette mit Willi Münzenberg zusammenlebte, war sie mit dem Wiener Journalisten Fritz Gross verheiratet.

87 Siehe zu Willi Münzenberg: Kap. II.3.1.

88 Buber-Neumann schildert in ihrem Buch Von Potsdam nach Moskau den Schock, den der Selbstmord von Heinz Neumanns Parteifreund Besso Lominadse - einem führenden Funktionär der Komintern, der trotz »Selbstkritik« 1934 aus der Partei ausgeschlossen wurde und der sich nach der Ermordung von Kirow erschoss, um der drohenden Verhaftung zu entgehen - bei ihr und Neumann verursachte (S. 394 f.). Auch durch diese totalitären Erfahrungen dachte Heinz Neumann angesichts der drohenden Verhaftung zwischenzeitlich immer wieder an Selbstmord (S. 429).

89 Margarete Buber-Neumann, Als Gefangene bei Stalin und Hitler. Eine Welt im Dunkel, Herford 1985, S. 18.

90 Reinhard Müller, Menschenfalle Moskau. Exil und stalinistische Verfolgung, Hamburg 2001. 
wurde, um die totale Treue gegenüber der Partei und dem Staat zu signalisieren. In diesem gesellschaftlichen Mikrokosmos »terrorisierter und atomisierter Individuen ${ }^{91}$ wurden unter dem Vorzeichen der »bolschewistischen Wachsamkeit« alte Rechnungen beglichen und frühere "Abweichler« von der offiziellen Parteilinie und "politisch unzuverlässige« an das NKWD ausgeliefert. Um das eigene Leben zu retten, wurde der Feind in den eigenen Reihen gesucht und Parteigenossen an das NKWD verraten. Unter der Herrschaft der allgegenwärtigen sowjetischen Geheimpolizei verstärkte die weitverbreitete Denunziationspraxis die Atmosphäre der Angst unter den »Politemigranten.$^{92}$ Jeder musste damit rechnen, vom anderen »ans Messer « geliefert $\mathrm{zu}$ werden, sodass sich schließlich die panische Angst ${ }^{93}$ vor einer möglichen Verhaftung immer mehr ausweitete und in vielen Fällen mit einer Wachsamkeitsparanoia korrespondierte..$^{4}$ Die totalitären Bedingungen während der stalinistischen Säuberungen führten zur »Atomisierung« der terrorisierten Subjekte und zum Abbruch der

91 Arendt, Elemente und Ursprünge totaler Herrschaft, S. 522.

92 Siehe zu der sich angesichts der stalinistischen Säuberung und der seit dem ersten Moskauer Schauprozesses im August 1936 um sich greifenden Atmosphäre der Angst unter den ausländischen Kommunisten insgesamt die konkreten Erfahrungen von Maragarete Buber-Neumann in dem Kapitel »Endstation Moskau«ihres Buches Von Potsdam nach Moskau (S. 393-444.)

93 In seinem autobiografischen Erfahrungsbuch beschrieb im Nachhinein der ehemalige Kommunist Wolfgang Leonhard die unter den deutschen »Politemigranten« und Kominterfunktionären grassierende Angst: »Die meisten waren von einer ständigen Angstpsychose befallen; sie liefen wie gehetztes Wild umher, immer darauf bedacht, nur ja das Richtige zu tun, um einer Verhaftung zu entgehen.«(Die Revolution entläßt ihre Kinder, Köln 1955, S. 39) - Vgl. hierzu die Rezension von Fritz René Allemann, Der junge Funktionär. Zu Wolfgang Leonhards Autobiografie. In: Der Monat 8 (1956), H. 90 , S. 58-60.

94 Eindrücklich schilderte der deutsche »Politemigrant« und damals führende Funktionär der KPD Herbert Wehner diese seit 1936 eintretenden atmosphärischen Bedingungen in seinen 1946 verfassten autobiografischen Notizen: »[1]m Gebäude der Komintern, in den Korridoren des Hotel Lux breitete sich damals ein panischer Schrecken, eine hysterische Angst vor einer ungreifbaren und doch so gut wie unentrinnbaren Gefahr aus. Wenn im Büro einer der Mitarbeiter nicht zur Arbeit erschienen war, nahmen seine Kollegen an, er sei in der Nacht durch die >Organe des NKWD < verhaftet worden. Sofort ergaben sich für jeden einzelnen zahllose Fragen: Wie wird das Verhältnis des Verhafteten zu mir vom NKWD ausgelegt werden? fragte sich wahrscheinlich jeder im Stillen. Äußerlich aber war jeder bestrebt, entweder unberührt zu erscheinen oder zu zeigen, daß er diese Verhaftung seit langem erwartet habe. Niemand wollte engere persönliche Beziehungen zu einem Verhafteten gehabt haben. Und weil in den sogenannten Parteiversammlungen der Abteilungen und des gesamten Apparats die persönlichen Verhältnisse und Beziehungen jedes Einzelnen schonungslos und schamlos ausgebreitet, nachträglich bewertet und zu Gegenständen wochenlanger Diskussion gemacht wurden, waren alle bestrebt, ihre persönlichen Beziehungen zu anderen auf das notwendige Minimum zu beschränken. Jeder war bestrebt sich von den meisten anderen in einem sicheren Abstand zu halten« (Herbert Wehner, Zeugnis. Köln 1982, S. 189 f.). Zum »inszenierten«Erinnerungsgehalt der »Notizen«, in denen sich Wehner mit Blick auf den »Großen Terror« als ohnmächtiges Opfer stilisierte, vgl. Reinhard Müller, Herbert Wehner - Moskau 1937, Hamburg 2004, wo gezeigt wird, dass der überzeugte »Trotzkistenjäger« eher als aktiver Mittäter im stalinistischen Terrorsystem zu sehen ist. Vgl. zu Wehner auch die Beiträge im Monat: Klaus Harpprecht, Lutheraner - halb links. Ein Porträt Herbert Wehners, in: Der Monat 19 (1967), H. 220 S. 13-17, sowie Günter Gaus, Der Schwierige - Versuch über Herbert Wehner, in: Der Monat 21 (1969), H. 244, S. 50-60. 
zwischenmenschlichen Beziehungen, ${ }^{95}$ sodass dies nicht nur für Buber-Neumann den »sozialen Tod« bedeutete.

Nach der Verhaftung von Neumann im April $1937^{96}$ rechnete in der Folgezeit im Moskauer »Lux « auch Buber-Neumann täglich mit ihrer Verhaftung, die dann im Juni 1938 erfolgte. Sie wurde beschuldigt, Mitglied einer oppositionellen Fraktion innerhalb der KPD gewesen zu sein und während dieser Zeit freundschaftlichen Kontakt zu bereits verhafteten deutschen Kommunisten gehabt zu haben. ${ }^{97}$ Ohne Gerichtsverfahren wurde sie zu fünf Jahren Konzentrationslagerhaft verurteilt. Nach einem mehrwöchigen Gefangenentransport kam sie mit mehreren Hunderten von Mithäftlingen in das riesige sibirische Lager von Karaganda. Dort mussten Buber-Neumann und die schätzungsweise 170000 Mitgefangenen unter extremsten Bedingungen in der Landwirtschaft und in den Kohlengruben arbeiten, wobei sie beispielsweise in den Sommermonaten täglich zwischen 14 und 15 Stunden im Einsatz waren. Aus jedem einzelnen Gefangenen holte die Lagerverwaltung bei minimaler Ernährung eine maximale

95 Der österreichische Physiker Alexander Weißberg-Cybulski (1901-1964) verglich diese Situation angesichts immer neuer Verhaftungen mit der Atmosphäre in Moskau insgesamt, in der es bereits gefährlich war, überhaupt Freunde zu haben, weil bei einer möglichen Verhaftung man selber in Bedrängnis käme, so: »Die Leute in Moskau zogen daraus die Konsequenz, jeden persönlichen Verkehr aufzugeben, sich in keinerlei Gespräch einzulassen, auf keinerlei Fragen zu antworten, sich auf die Arbeit und die nächste Familie zu beschränken.« (Im Verhör. Ein Überlebender der stalinistischen Säuberungen berichtet, Wien 1993, S. 73)

96 In ihren autobiografischen Erinnerungen berichtete Margarete Buber-Neumann, dass es in den Jahren des stalinistischen Terrors zu einer typischen perfiden Herrschaftsmethode der verschiedenen NKWD-, Gefängnis- und Staatsanwaltschaftsinstanzen zählte, den Verwandten Nachrichten über das Schicksal von Verhafteten vorzuenthalten und sie zum Teil jahrelang im Ungewissen zu lassen, sodass sich die mit eben dieser Verhaftung verbundenen Schrecken noch vergrößerten (Als Gefangene bei Stalin und Hitler, S. 24 f.). Buber-Neumann erfuhr erst nach dem Zweiten Weltkrieg von den sowjetischen Behörden, dass Neumann in der Sowjetunion ums Leben kam, ohne genaue Angaben über Datum und Ursache des Todes. Tatsächlich wurde er jedoch am 26. November 1937 zum Tode verurteilt und anschließend durch Genickschuss ermordet.

97 Das Kontaktschuldprinzip war ein immer wiederkehrender Anklagepunkt in den Jahren des stalinistischen Terrors und hatte fatale Konsequenzen auch und vor allem für die im Moskauer Exil ausweglos festgesetzten Emigranten, denn es führte in dieser Personengruppe-wie gezeigt-zu einem Klima der Angst und des gesteigerten Verdachts und gehörte zu den gemachten Erfahrungen vieler verfolgter Kommunisten. Hannah Arendt sprach in diesem Zusammenhang im Hinblick auf die atomisierten und isolierten Individuen im Moskauer Exil von einer »Präparierung der Opfer«, die »den einzelnen gleich gut für die Rolle des Vollstreckers wie für die des Opfers vorbereiten kann« (Elemente und Ursprünge totaler Herrschaft, S. 716). Denn das Prinzip »guilt by association «, das Arendt als »zentrales Konstituens der Sowjetgesellschaft« ansah, implizierte: »Sobald gegen jemanden Anklage erhoben wird, müssen sich seine Freunde über Nacht in seine erbittertsten und gefährlichsten Feinde verwandeln, weil sie dadurch, daß sie ihn denunzieren und dabei helfen, das Aktenstück der Polizei und der Staatsanwaltschaft gehörig anzureichern, sich ihrer eigenen Haut wehren können; da es sich bei den Anklagen im allgemeinen um nichtexistente Verbrechen handelt, braucht man gerade sie, um den Indizienbeweis zu erbringen. Während der großen Säuberungswellen gibt es überhaupt nur ein Mittel, die eigene Zuverlässigkeit zu beweisen, und das ist die Denunziation seiner Freunde. Und dies wiederum ist, was die totale Herrschaft und die Mitgliedschaft in einer totalitären Bewegung angeht, ein durchaus richtiger Maßstab; hier ist in der Tat nur der zuverlässig, der seine Freunde zu verraten bereit ist. Was suspekt ist, ist Freundschaft und jegliche andere menschliche Bindung überhaupt.«(Ebd., S. 523 f.) 
Arbeitsleistung heraus, sodass dies bei erbärmlichen Unterbringungsbedingungen in vielen Fällen den sicheren Tod bedeutete. Buber-Neumann überlebte die Lagerhaft nicht zuletzt deshalb, weil sie nach einer sehr schweren Krankheit von dem Lagerarzt, einem Mithäftling, von extremer schwerer Arbeit befreit wurde. ${ }^{98}$

Allerdings waren diese extremen Arbeits- und Lebensbedingungen nur die eine Seite des Alltags im Lager. $\mathrm{Zu}$ den totalitären Erfahrungen von Buber-Neumann in Karaganda gehörte auch, dass das NKWD bewusst »kriminelle Gefangene« zur Herrschaftssicherung und -verwaltung im Lager einsetzte. So entstand für die sogenannten politischen, religiösen und asozialen Häftlinge eine zweite Herrschaftsgruppe, deren fast willkürlichen Maßnahmen sie ausgesetzt waren, die von einzelnen Schikanen über Diebstahl bis zu Mord reichten. ${ }^{99}$

Als sich Buber-Neumann mit ihrem Gefangenendasein im sibirischen Lager in Karaganda abgefunden hatte und jede Hoffnung auf eine Freilassung bei ihr bereits erloschen war, trat völlig überraschend ein Wendepunkt ein. Nachdem der Zweite Weltkrieg ausgebrochen war, wurde sie Anfang 1940 mit zwei weiteren Frauen aus Deutschland in ein Untersuchungsgefängnis nach Moskau gebracht. Sie trafen dort auf mehrere Frauen aus Deutschland und Österreich - unter anderem Zenzl Mühsam und Carola Neher -, die aus sowjetischen Lagern und Zuchthäusern kamen und die, wie Buber-Neumann, alle vor dem Nationalsozialismus in die Sowjetunion geflüchtet waren. Auch aufgrund der ganz besonderen Haftsituation - es gab genügend Ernährung, die Frauen konnten heiß baden, wurden neu eingekleidet und »in jede Richtung umsorgt « - keimte bei Buber-Neumann die große Hoffnung auf, »über die litauische Grenze, in das Ausland abgeschoben zu werden ${ }^{100}$.

Dies sollte sich indes als Illusion erweisen. Denn es kam vollkommen anders. Mit insgesamt 30 weiteren deutschen und österreichischen Gefangenen wurde BuberNeumann unter Bewachung von Soldaten und einem NKWD-Offizier mit der Eisenbahn nach Brest-Litowsk gebracht. Genauer gesagt, die Gruppe wurde zur Brücke gebracht, die über den Fluss Bug führte, der im Jahre 1940 zum Zeitpunkt des Hitler-Stalin-Paktes die Demarkationslinie zwischen dem von Deutschland und dem von der Sowjetunion besetzten Polen war. Was sie auf der Eisenbahnbrücke erlebte, was einem traumatischen Schock gleichkam, schilderte Buber-Neumann an einer zentralen Stelle ihres Buches mit folgenden Worten:

Alle Gesichter waren gleich starr vor Angst. Wir standen und blickten über diese Eisenbahnbrücke, die die Grenze bildete zwischen dem von den Deutschen besetzten Polen

98 Siehe hierzu im Einzelnen Buber-Neumann, Als Gefangene bei Stalin und Hitler, S. 132-138.

99 Buber-Neumann, die als »politische Cefangene« im Lager inhaftiert war, schrieb in ihrem Buch über diese Häftlingsaristokratie: »Die Kriminellen sind in Sibirien die Häftlingsgattung, der es am besten geht. Sie haben die einträglichsten Posten inne, halten fest zusammen und sind, man könnte fast sagen, organisiert. Für sie bedeutet die Haft keine Unterbrechung ihres gewohnten Daseins. Das Gefängnis gehört zu ihren Berufsrisiken. Verfügt der Führer der Kriminellen zum Beispiel, daß am nächsten Tage nicht gearbeitet wird, so wagt keiner seiner Untertanen, gegen seinen Befehl zu handeln, obgleich nach der Lagerordnung von Karaganda auf fünfundzwanzig Arbeitsverweigerungen die Todesstrafe stand. Die Kriminellen blicken voller Verachtung auf die Politischen herab, auf die Feinde der Sowjetunion, während sie stolz darauf sind, zwar Kriminelle, aber doch gute Sowjetbürger zu sein.«(Ebd., S. 79)

100 Ebd., S. 178. 
und dem von den Russen okkupierten Teil. Über die Brücke ging ein Soldat langsam auf uns zu. Als er näherkam, erkannte ich die Soldatenmütze der SS. Der NKWD-Offizier und der von der SS hoben grüßend die Hand an die Mütze. Aus einer hellbraunen, länglichen Tasche zog der NKWD-Offizier eine Liste. Er war fast um einen Kopf größer als der SS-Mann. Sein Gesicht war ledern und maskenhaft, wie es in Schundromanen immer heißt. Welchen Namen er herunterlas, hörte ich gar nicht. Irgendwann vernahm ich »Buber-Nejman«, und da sah ich, wie sich drei von unserer Gruppe absonderten und erregt mit dem NKWD-Offizier sprachen. Irgendwer flüsterte: »Die weigern sich über die Brücke zu gehen!« [...] Dann sah ich, wie die drei über die Brücke getrieben wurde. Auf den ungarischen Emigranten, der einen Koffer trug, hatte es der SS-Mann besonders abgesehen: »Das jüdische Schwein will wohl kommunistische Literatur nach Deutschland einschmuggeln!? Dem werden wir die Hammelwaden noch langziehen! Schneller! Schneller! Nur keine Müdigkeit vorschützen! «101

Für Buber-Neumann stand zweifelsohne fest, dass für die mit Gewalt über die Brücke getriebenen und den Nazis übergebenen drei Gefangenen - neben dem ungarischen Juden handelte es sich hier noch um einen Lehrer aus Mitteldeutschland sowie um einen von der Gestapo in Abwesenheit zum Tode verurteilten jungen kommunistischen Arbeiter aus Dresden - auf der anderen Seite des Flusses der sichere Tod wartete. ${ }^{102} \mathrm{Zu}$ ihrer persönlichen Auslieferung an die Nazis schrieb Buber-Neumann weiter:

Auf der anderen Seite der Brest-Litowsker Brücke stand eine Holzbude. Betty Olberg [eine gehbehinderte kommunistische Mitgefangene] schwankte vor Schwäche, Kälte und Erregung. Einer schlug vor: »Bringt sie doch in die Hütte!« Man ließ uns beide hinein. Ein SS-Mann mit einem Polizeihund neben sich öffnete die Tür. Da sah ich das erstemal so ganz in der Nähe die SS-Mütze mit dem Totenkopf und gekreuzten Knochen und eine richtige SS-Visage darunter. »Setzen Sie sich!« forderte er uns im Befehlston auf. $^{103}$

Buber-Neumann musste zahlreiche Verhöre durch die Gestapo in einem Berliner Untersuchungsgefängnis über sich ergehen lassen. Da nach der Einschätzung der Gestapo das Anklagematerial für ein Gerichtsverfahren nicht ausreichte, indes der Verdacht bestand, dass sie sich für die verbotene KPD betätigen wird, stellte die nationalsozialistische Behörde einen sogenannten Schutzhaftschein gegen sie aus. Anfang August 1940 wurde Buber-Neumann in das Frauenkonzentrationslager Ravensbrück in Mecklenburg eingeliefert. Über den Schock, den die unbefristete Verurteilung in das nationalsozialistische Lager bei ihr verursachte, stellt Buber-Neumann in ihrem autobiografischen Erfahrungsbuch fest:

Nach der Aushändigung des Schutzhaftscheines waren meine Kräfte am Versagen. Wieder Konzentrationslager! Eben dem sicheren Tod in Sibirien entronnen, und nun in eine neue Hölle! Es ist so viel einfacher, einem unbekannten Schicksal entgegenzuge-

101 Ebd., S. $179 \mathrm{f}$.

102 Margarete Buber Neumann, Als Gefangene bei Stalin und Hitler [undatierter Vortrag], in: Janine Platten/Judith Buber-Agassi (Hg.), Margarete Buber-Neumann. Plädoyer für Freiheit und Menschlichkeit. Vorträge aus 35 Jahren, Berlin 2000, S. 110-116, hier S. 114.

103 Buber-Neumann, Als Gefangene bei Stalin und Hitler, S. 180. 
hen, aber ich wußte doch nur zu gut, was Konzentrationslager bedeutet. Alle um mich herum redeten von »drei Monaten Umschulung«, aber ich hatte keine Spur von Hoffnung auf eine baldige Befreiung. Und trotzdem lebte man weiter. ${ }^{104}$

Die schrecklichen Erfahrungen von Karaganda wiederholten sich in den Augen von Buber-Neumann im nationalsozialistischen Lager von Ravensbrück. Sie wurde zu Beginn mit einigen tausend Frauen zusammengesperrt und erlebte von Anbeginn den Terror der SS-Bewachung. Neben der alltäglichen Gewalt und der nach ihrer Einschätzung ab 1942 einsetzenden systematischen Ermordung von Häftlingen entwickelte sich für Buber-Neumann der >Arbeitseinsatz« zur "gefürchtetsten Institution von Ravensbrück «. ${ }^{105}$ Diese schrecklichen Erfahrungen wurden noch gesteigert, als sich im Laufe des Zweiten Weltkrieges das Lager auf bis zu knapp 20000 Frauen füllte, sodass dies zu einer Überfüllung der Baracken führte und für Buber-Neumann das Leben in Ravensbrück »zu einer Hölle« machte. Denn in den letzten Jahren mussten sich beispielsweise drei Frauen einen Strohsack teilen und die große Anzahl von Gefangenen führte dazu, dass sich Ungeziefer und Hunger immer mehr ausbreiteten und die Kanalisation dauernd übertrat, "so daß zwischen den Baracken im Sommer ein Kloakensee schwamm, der im Winter zu einer Eisenbahn wurde $«^{106}$.

$\mathrm{Zu}$ den in Ravensbrück gemachten totalitären Erfahrungen gehörte für BuberNeumann auch, dass sie den nationalsozialistischen Terror gegenüber anderen Gefangenen miterlebte. Sie bekam unter anderem die schrecklichen medizinischen Experimente an zumeist polnischen Häftlingen mit, die entweder daran starben oder gezwungen waren, mit den ihnen zugefügten Verstümmelungen im Lager weiter zu leben und zu arbeiten. Frühzeitig erfuhr Buber-Neumann von einem Transport von Alten, Kranken und jüdischen Frauen in das Konzentrationslager Bernburg bei Dessau, wo sie durch das Giftgas Zyklon B umgebracht wurden. Zudem erlebte sie ab Sommer 1942 die regelmäßigen Transporte von Juden, »Zigeunern« und »lebensunwerten Leben«, d. h. von alten und kranken Mitgefangenen, die nicht mehr »arbeitsfähig« waren, von Ravensbrück nach Auschwitz, wo sie vergast wurden. Im Laufe des Jahres 1944 verwandelte sich Ravensbrück in ein Vernichtungslager, wo fortan Buber-Neumann miterlebte, wie die SS alle nicht mehr als »arbeitsfähig« eingestuften Frauen in der Gaskammer umbrachten. Noch wenige Monate vor dem Ende des Zweiten Weltkrieges wurden in Ravensbrück 4000 Frauen vergast. ${ }^{107}$

Neben den existenziellen Erfahrungen, die Buber-Neumann mit dem allgegenwärtigen nationalsozialistischen Terror im Konzentrationslager Ravensbrück machte und der dazu führte, dass sie zweimal nur knapp dem Tod entging, existierte noch eine »zweite Terrorrealität«. Mit Erschrecken musste sie feststellen, dass sie vonseiten der in Ravensbrück inhaftierten Kommunistinnen verfolgt, terrorisiert und wie eine Aussätzige behandelt wurde. Zum Beispiel erlebte sie bereits am dritten Tag »voller Erstaunen« im Aufnahmeblock eine Verhörsituation von drei deutschen Kommunistinnen, in der Buber-Neumann gezwungen wurde, über ihre Erfahrungen in der Sowjetunion zu berichten und die genauen Umstände der Verhaftung durch die Ge-

104 Ebd., S. 206.

105 Ebd., S. 334.

106 Ebd., S. 231.

107 Ebd., S. 362. 
stapo zu schildern. Noch vor dem eigentlichen »Eintritt in die Lageröffentlichkeit«, so Buber-Neumann, musste sie erfahren, dass sie »bereits geächtet war ${ }^{108}$ und fortan mit dem Stigma der »Verräterin« bzw. »Trotzkistin« fünf Jahre lang bis zu ihrer Befreiung unter den kommunistischen Mitgefangenen im Konzentrationslager Ravensbrück leben musste. ${ }^{109}$

Margarete Buber-Neumanns antitotalitäres Engagement nach dem Ende des Zweiten Weltkrieges war den totalitären Erfahrungen in den Jahren des »Großen Terrors « in Moskau, in dem sowjetischen Arbeitslager in Karaganda und in dem nationalsozialistischen Konzentrationslager Ravensbrück geschuldet. Ihren Antitotalitarismus begründete sie in einem Vortrag mit dem Titel Als Gefangene bei Stalin und Hitler mit den persönlich erlebten Schrecken und führte hierzu aus:

Die Hitlerbarbarei gehört der Vergangenheit an; in den kommunistischen Diktaturen hingegen leiden bis zum heutigen Tage Abermillionen Menschen unter Terror und Not. Diese Tatsache bringt mich dazu, immer wieder über die Verbrechen der beiden großen Diktaturen unserer Zeit zu sprechen, denn das, was dort geschah und heute noch geschieht, kann der Mensch, der es nicht erlebte, kaum ermessen. ${ }^{110}$

\section{Frühe Auseinandersetzung der Renegaten mit dem Totalitarismus vor dem Hintergrund ihrer Rolle im Monat}

\subsection{Die Auseinandersetzung mit dem Totalitarismus in den Zeitschriftenprojekten von Willi Münzenberg und Dwight Macdonald}

Wie gezeigt, setzte die Auseinandersetzung mit den totalitären Herrschaftsregimen aufseiten zahlreicher Autoren des Monat keineswegs erst mit dem beginnenden Kalten Krieg ein. Es waren unter anderem ehemalige Anhänger der kommunistischen Bewegung, die zu einem wesentlich früheren Zeitpunkt die politische und theoretische

108 Ebd., S. 218.

109 Hierzu stellte Buber-Neumanns Tochter Judith Buber-Agassi im Nachhinein fest: »Nur die Tatsache, daß sie sich so viele treue Freundinnen unter den nichtkommunistischen deutschen, französischen, norwegischen und tschechischen Häftlingen erwarb, und daß in einigen seltenen Fällen ein kommunistischer Häftling gegen die Feme rebellierte, rettete ihr das Leben.« (Judith Buber-Agassi, Margarete Buber-Neumann. Ein biographischer Essay, in: Janine Platten/Dies. (Hg.), Margarete Buber-Neumann. Plädoyer für Freiheit und Menschlichkeit. Vorträge aus 35 Jahren, Berlin 2000, S. 10-30, hier S. 18) - Zur wichtigsten Freundin im Konzentrationslager Ravensbrück avancierte die anerkannte tschechische Journalistin Milena Jesenska. Die zwischenzeitliche Freundin von Franz Kafka brach anläßlich der Moskauer Schauprozesse mit der Kommunistischen Partei. Sie zählte zum tschechischen Widerstand gegen den Nationalsozialismus und zeichnete mitverantwortlich für die Rettung zahlreicher tschechischer Juden und Landsleute, denen mit ihrer Hilfe die Flucht ins Ausland gelang. Jesenska glaubte von Anbeginn dem autobiografischen Erfahrungsbericht der als »Trotzkistin«stigmatisierten deutschen Kommunistin Buber-Neumann über das Lagersystem in der Sowjetunion. Sie starb 1944 im Konzentrationslager Ravensbrück. Zur Ceschichte der Freundschaft zu Milena Jesenska vgl. Margarete Buber-Neumann, Milena, Kafkas Freundin, München 1963.

110 Buber-Neumann, Als Gefangene bei Stalin und Hitler [undat. Vortrag], S. 116. 